\title{
Reconstruction Parameters of Local Scattering Sources of a Metallic Strip from the Backscattering Pattern
}

\author{
Stanislav N. Kutishchev \\ Department of Physics and Chemistry, Voronezh State University of Architecture and Civil Engineering, Voronezh, Russia. \\ Email: kutich@list.ru
}

Received October $11^{\text {th }}, 2012$; revised November $13^{\text {th }}, 2012$; accepted November $23^{\text {rd }}, 2012$

\begin{abstract}
In this paper, it is proved the ability of quantity reconstruction, amplitudes and coordinates of metallic strip local scattering sources from the backscattering pattern. They are performed as the results of numerical solution for the infinite perfect conducting strip in case of E-polarization of the incident plane electromagnetic wave. In this case it is necessary to fulfill the following conditions. The local sources amplitudes should be the same order, in transverse and longitudinal directions the local sources should be separated into distances more than apparatus resolution, and the object maximum size does not have to be more than approximately $50 \lambda$. It was shown the limit and ability of the further development of the offered method.
\end{abstract}

Keywords: Backscattering Pattern; Local Scattering Sources; Radar Image; Electromagnetic Wave; Method; Metallic Strip

\section{Introduction}

Nowadays the researches of creating of objects scattering structure reconstruction methods in scattering field in radiolocation [1-7], antenna theory [8], radio astronomy [9], optics [10] and other scientific regions are being done. Urgency of radiolocation work in this region is brought about, for example, the essential of getting rather full information about space structure of local scattering sources [11] on the surface of complex shape objects with the purpose of improving means and methods of the objects radar visibility decreasing.

Doing radiolocation characteristic research of objects at ranges and in laboratories the scattering pattern is built as a result of the object turning. So reconstruction problem of local scattering sources of this object appears from the backscattering pattern. For the object model [12] as the system of isotropic rigidly tied and electrodynamically independent scatters in work [7] the method of numerical solution of the problems was offered. It is interesting to use this method for the solution of analyzing problem in case of the infinite (along $\mathrm{z}$ axis) perfect conducting strip (Figure 1). The strip is believed to be infinitely thin.

Work aim is the researching of the reconstruction ability of parameters (quantity, amplitudes and coordinates) of metallic strip scattering sources from the backscattering pattern.

The reconstruction ability of the parameters of the metallic cylindrical object (metallic strip) local scattering sources has been first investigated in the paper. The solution of the inverse electrodynamical problem is based on apriori assumption about discretion of local sources the cylindrical object (the model of isotropic rigidly tied and electrodynamically independent scatters). The dependences of the reconstructed amplitudes and transverse coordinates of two local sources of the metallic strip on the aspect angle (the case of a monostatic scattering) have been first numerically obtained. As the result of analysis of the obtained numerical data it was found that the absolute error of the reconstruction of the transverse coordinates of the local sources does not exceed approximately $0.03 \lambda$. The limits of the offered method were estimated.

\section{Method of Reconstruction}

Let's look at the case of a monostatic scattering of the plane electromagnetic wave from the system of $\mathrm{N}$ isotropic rigidly tied and electrodynamically independent scatters (Figure 2) which is the electrodynamical model [12] of the object. Receiving narrow-band reflected signal in the far zone of the object and transmit-receive antenna the backscattering pattern including the results of work [2] and the problem geometry (believing that measurements are done in $x y$-plane) can be performed as:

$$
E^{(0)}(\varphi)=\sum_{i=1}^{N} E_{i} \exp \left[j 2 k\left(x_{i} \cos \varphi+y_{i} \sin \varphi\right)\right]
$$




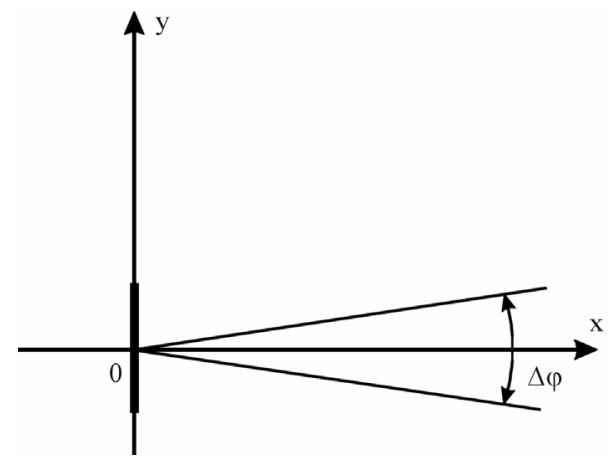

Figure 1. Metallic strip.

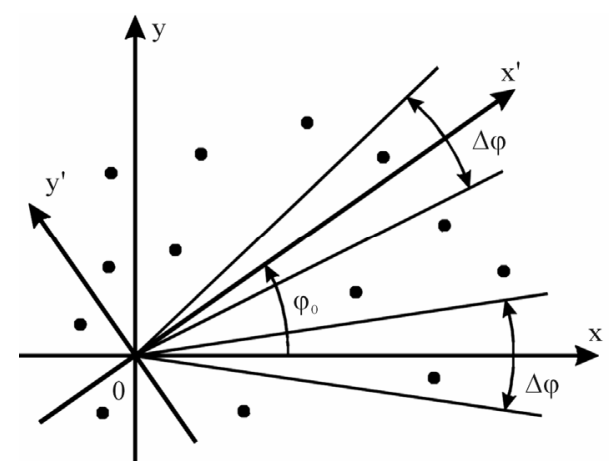

Figure 2. Object model.

where $x_{m}, y_{m}$ are the coordinates of m-scatter; $E_{m}$ is the amplitude of the signal scattering from m-scatter; $\varphi$ is the observation angle calculated from $\mathrm{x}$-axis directed along bisector of angles $\Delta \varphi ; k=2 \pi / \lambda$ is the wave number; $\lambda$ is the wavelength. Formula (1) is right for every observation angles $\varphi$.

When $\Delta \varphi / 2 \ll 1$ formula (1) can be linearized and changed as:

$$
E^{(0)}(u)=\sum_{i=1}^{N} \tilde{E}_{i} \exp \left(j 2 y_{i} u\right),|u| \leq k \sin (\Delta \varphi / 2) \ll k,
$$

where $\tilde{E}_{n}=E_{n} \exp \left(j 2 k x_{n}\right), u=k \sin (\varphi)$-spatial frequency.

So this problem can be expressed as: for observing object model is necessary to find the number of local scattering sources $\mathrm{N}$, their amplitudes $E_{i}$ and their transversal and longitudinal coordinates $y_{i}$ and $x_{i}$ according to the known from experiment backscattering pattern $E^{(0)}(u)$, where $|u| \leq k \sin (\Delta \varphi / 2) \ll k$.

The method of the solution of the considered problem consists of some stages [7].

Stage 1. From fragment $E^{(0)}(\varphi)$, which is known at $|\varphi| \leq \Delta \varphi / 2 \ll 1$, quantity of local sources $\mathrm{N}$, their transverse coordinates $y_{i}$ and amplitudes are found. Namely, spatial frequency spectrum $E(u, \Delta \varphi)$ is found out from the fragment known from measurements backscattering pattern of the object $E^{(0)}(\varphi)(1)$ :

$$
E(u, \Delta \varphi)= \begin{cases}E^{(0)}(u), & |u| \leq k \sin (\Delta \varphi / 2) \ll k, \\ 0, & |u|>k \sin (\Delta \varphi / 2) .\end{cases}
$$

Writing the spatial frequency spectrum $E(u, \Delta \varphi)$ in (3) a rectangular window [13] is used, legal using of which is connected with the small value of observation angles sector $\Delta \varphi$.

Using spatial frequency spectrum $E(u, \Delta \varphi)$ one-dimensional radar image $J(y, \Delta \varphi)$ of the object [3-7,14] is found:

$$
\begin{aligned}
J(y, \Delta \varphi) & =\frac{1}{2 \pi} \int_{-\infty}^{\infty} E(u, \Delta \varphi) \exp (-j y u) \mathrm{d} u \\
& =\frac{k \Delta \varphi}{2 \pi} \sum_{i=1}^{N} \tilde{E}_{i} \frac{\sin \left(\frac{k \Delta \varphi}{2}\left(y-2 y_{i}\right)\right)}{\frac{k \Delta \varphi}{2}\left(y-2 y_{i}\right)},
\end{aligned}
$$

From this formula it is known that the number of main maxima equals to the number of local sources $\mathrm{N}$. The transversal coordinates of the local sources equal a half of the transverse ones going with main maxima. The values of the main maxima of the one-dimensional radar image $J(y, \Delta \varphi)$ (4) correspond to the amplitudes $E_{i}$ of the object local sources.

Stage 2. Using the fragment $E^{(0)}(\varphi)$ which is known at $\left|\varphi-\varphi_{0}\right| \leq \Delta \varphi / 2 \ll 1$, with algorithm help of the 1 st stage the transverse coordinates $y_{i}^{\prime}$ of the local sources in $x^{\prime} y^{\prime}$ coordinates system turned to the angle $\varphi_{0}$ with respect to $x y$ coordinates system (Figure 2) are calculated.

Let us look at the backscattering pattern $E^{(0)}(\varphi)$ in the aspect angles sector $\left|\varphi-\varphi_{0}\right| \leq \Delta \varphi / 2 \ll 1 \quad$ (Figure 2). Using the well-known relations [15] for the coordinate transformation of the local scattering sources let us write it in $x^{\prime} y^{\prime}$ coordinates system as [4]:

$$
\begin{aligned}
& E^{(0)}(\varphi) \\
& =\sum_{i=1}^{N} E_{i} \exp \left[j 2 k\left(x_{i}^{\prime} \cos \left(\varphi-\varphi_{0}\right)+y_{i}^{\prime} \sin \left(\varphi-\varphi_{0}\right)\right)\right], \\
& \left|\varphi-\varphi_{0}\right| \leq \Delta \varphi / 2 \ll 1 .
\end{aligned}
$$

In this situation $\varphi_{0}$ can take any values.

From the match up (5) and (1) it is summed that the algorithm of calculation of the transverse coordinates $y_{i}^{\prime}$ of the second stage is analogous to the algorithm of calculation of the transverse coordinates $y_{i}$ of the first stage.

From the resulting local sources transverse coordinates $y_{i}, y_{i}^{\prime}$ their longitudinal ones $y_{i}$ are calculated (Figure 2) $[4]$ :

$$
x_{i}=y_{i} \operatorname{ctg} \varphi_{0}(i=1, \cdots, N) .
$$

From formula (6) it is known that if we increase $\varphi_{0}$, an error of definition of the local sources longitudinal co- 
ordinates decreases. For $\varphi_{0}=90^{\circ}$

$$
x_{i}=-y_{i}^{\prime} .
$$

In this case the error of the reconstruction of the longitudinal coordinates in $x y$ system equals to the error of the reconstruction of the transverse coordinates in $x^{\prime} y^{\prime}$ system. So at the second stage it is better to choose $\varphi_{0}=90^{\circ}$.

\section{Numerical Results and Discussion}

Later there are the results of the solution of the observing problem for the metallic strip with the lower edge coordinates $(0,-5 \lambda)$ and upper edge coordinates $(0,5 \lambda)$ (Figure 1). The backscattering pattern of the strip was calculated by the rigorous method of the integral equations [16] in case of E-polarization ( $\boldsymbol{E}$ is directed along $\mathrm{z}$ axis) of the incident plane monochromatic electromagnetic wave with the amplitude equals 30 .

In Figure 3 the dependences of the reconstructed transverse coordinates of two local sources of the strip on the aspect angle $\varphi$ are shown. The value of the aspect angles sector $\Delta \varphi=12^{\circ}$. In this case two local sources starting with $\varphi_{0}=6^{\circ}$ are reconstructed when the normal angle to the strip surface is not thrown into the aspect angles sector $\left(\varphi_{0}=0^{\circ}\right)$. Curves 1 and 2 , Figure 3 practically equal, so the first local source is located on the lower edge of the strip. For the aspect angles $\varphi_{0}>48^{\circ}$ the amplitude of the first local source is extremely small (Figure 4) and this local source is not reconstructed. Curves 3 and 4, Figure 3 practically match, so the second local source is located on the strip upper edge for the aspect angles $\left(6^{\circ}, 90^{\circ}\right)$. The local scattering sources of the strip are located on its surface, so the reconstruction of its longitudinal coordinates is obvious.

In Figure 4 the dependences of reconstructed amplitudes of two local sources of the strip are shown on the

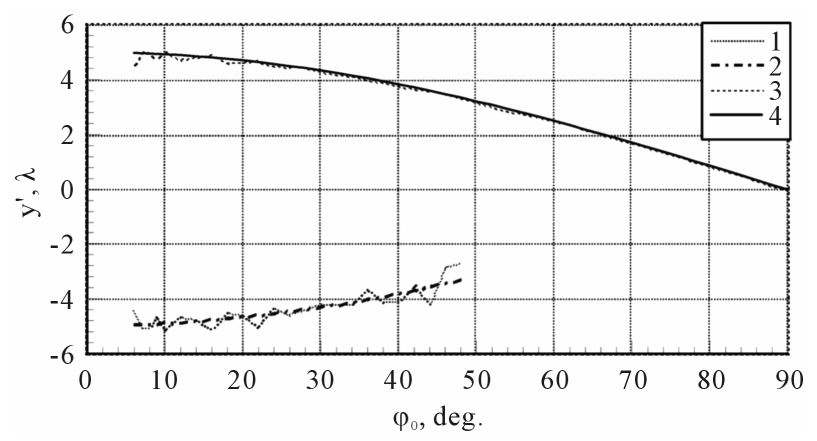

Figure 3. The dependence of the reconstructed transverse coordinates of two local sources of the strip on the aspect angle $\varphi_{0}(1)$ The reconstructed coordinates of the first local source; (2) Coordinates of the strip lower edge; (3) The reconstructed coordinates of the second local source; (4) Coordinates of the strip upper edge.

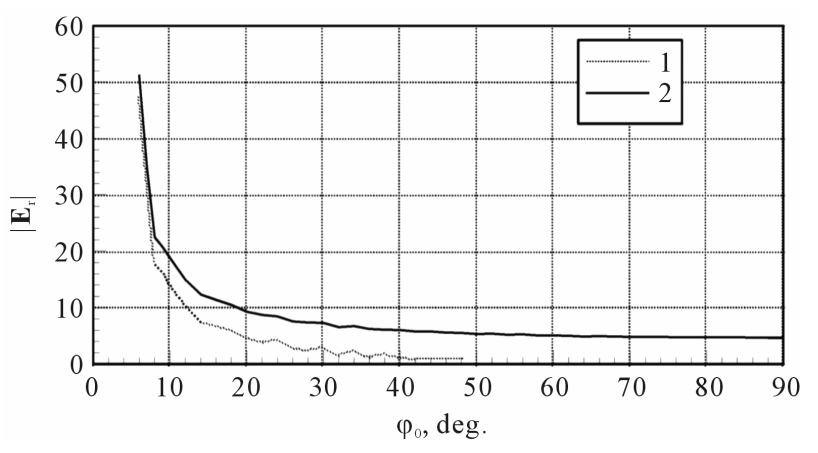

Figure 4. The dependence of the reconstructed amplitudes of two local sources of the strip on the aspect angle $\varphi_{0}$. (1) The first local source; (2) The second local source.

aspect angle $\varphi_{0}$. Their amplitudes are practically equal for small aspect angles. Increasing the observation angle $\varphi_{0}$ the amplitude of the second local source starts over the amplitude of the first local source. For the aspect angles $\varphi_{0}$ $>48^{\circ}$ the amplitude of the first local source becomes negligibly small, as the amplitude of the second one at $\varphi_{0}$ $=90^{\circ}$ equals 4.67 .

Further as an illustration the results of reconstruction of the local sources of this metallic strip for case $\Delta \varphi=12^{\circ}$ and $\varphi_{0}=28^{\circ}$ are shown.

The curve Figure 5 is a fragment of the amplitude backscattering pattern $|E(u)|$ of the strip in spatial frequency sector $u \in\left[k \sin \left(22^{\circ}\right) ; k \sin \left(34^{\circ}\right)\right],\left(\Delta \varphi=12^{\circ}, \varphi_{0}\right.$ $=28^{\circ}$ ).

The curve Figure 6 is a fragment of the phase backscattering pattern $\arg (u)$ (let us notice that $E(u)=|E(u)| \exp (j \arg (u)))$ of the strip in spatial frequency sector $u \in\left[k \sin \left(22^{\circ}\right) ; k \sin \left(34^{\circ}\right)\right]\left(\Delta \varphi=12^{\circ}, \varphi_{0}=\right.$ $\left.28^{\circ}\right)$.

In Figure 7 the modulus of the one-dimensional image of the strip $|J|$ for $u \in\left[k \sin \left(22^{\circ}\right) ; k \sin \left(34^{\circ}\right)\right]\left(\Delta \varphi=12^{\circ}\right.$, $\left.\varphi_{0}=28^{\circ}\right)$ is shown. Two main maxima correspond to two local sources $(\mathrm{N}=2)$. The reconstructed transverse coordinates of the local sources $y_{r}^{\prime}$ (Table 1) equal to a half of transverse coordinates of the corresponding main maxima. The transverse coordinates $y^{\prime}$ (Table 1) correspond to the transverse coordinates of the strip edges. The modulus values of the main maxima of the one-dimensional image $J(y, \Delta \varphi)$ correspond to the modulus of the reconstructed amplitudes of the local sources $\left|E_{r}\right|$ (Table 1).

As the result of analysis of the obtained numerical data (Figure 3) it was found that the absolute error of the reconstruction of the transverse coordinates of the local sources does not exceed approximately $0.03 \lambda$. The analysis of numerical results (Table 1) affords us to summarize that in the considered example $\left(\Delta \varphi=12^{\circ}, \varphi_{0}=28^{\circ}\right)$ the absolute error of the reconstruction of the transverse coordinates of the local sources $\Delta y \approx 0.03 \lambda$. 


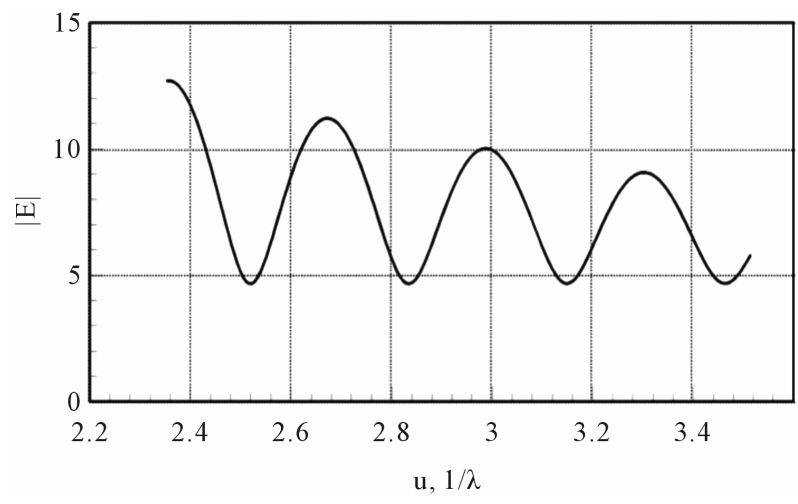

Figure 5. The amplitude backscattering pattern of the strip in spatial frequencies sector $u \in\left[k \sin \left(22^{\circ}\right) ; k \sin \left(34^{\circ}\right)\right](\Delta \varphi$ $=12^{\circ}, \varphi_{0}=28^{\circ}$ ).

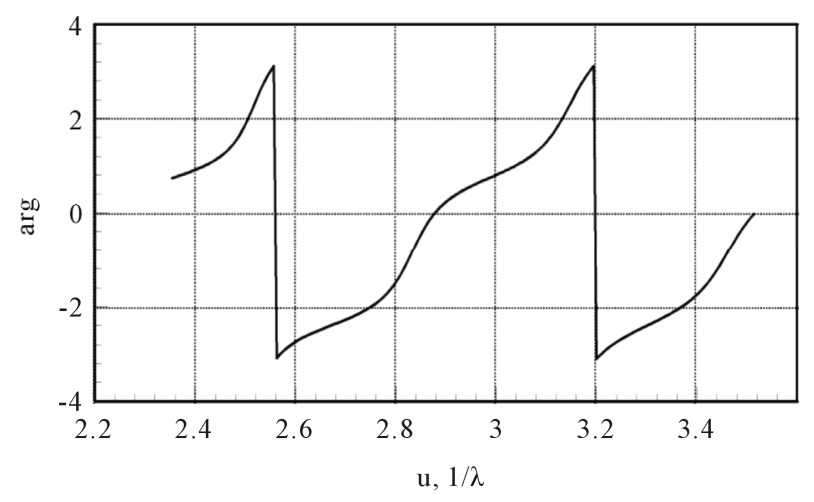

Figure 6. The phase backscattering pattern of the strip in spatial frequencies sector $u \in\left[k \sin \left(22^{\circ}\right) ; k \sin \left(34^{\circ}\right)\right](\Delta \varphi=$ $12^{\circ}, \varphi_{0}=28^{\circ}$ ).

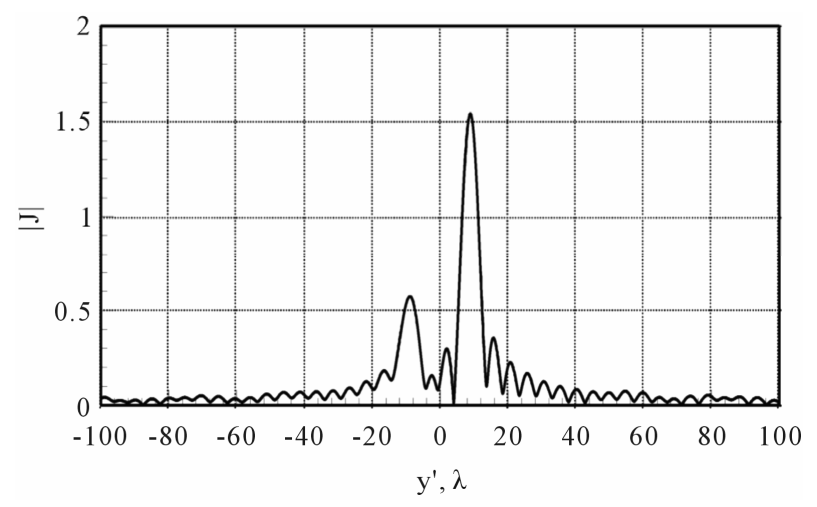

Figure 7. The modulus of the one-dimensional image $\mathbf{J}$ of the strip for $u \in\left[k \sin \left(22^{\circ}\right) ; k \sin \left(34^{\circ}\right)\right]\left(\Delta \varphi=12^{\circ}, \varphi_{0}=28^{\circ}\right)$.

Table 1. Data of the strip local scattering sources.

\begin{tabular}{cccc}
\hline$i$ & $y^{\prime}$ & $y_{r}^{\prime}$ & $\left|E_{r}\right|$ \\
\hline 1 & $-4.42 \lambda$ & $-4.45 \lambda$ & 2.74 \\
2 & $4.42 \lambda$ & $4.45 \lambda$ & 7.35 \\
\hline
\end{tabular}

\section{Conclusions}

As a result, this method allows us to reconstruct from backscattering pattern the parameters (quantity, amplitudes and coordinates) of isotropic stiff-tied and electrodynamically independent local scattering sources of a metallic strip. In this case it is necessary to fulfill the following conditions. The local sources amplitudes should be the same order, in transverse and longitudinal directions the local sources should be separated into distances more than resolution $\delta=\lambda /(2 \Delta \varphi)$, and the object maximum size does not have to be more than approximately $50 \lambda$.

Further it is planned to use this method for the reconstruction of the local scattering sources of two-dimensional cavities of a complex shape.

\section{REFERENCES}

[1] A. A. Luchin, "Methods of Approximate Solution of the Inverse Problem in Radiolocation," Zarubezhnaya Radioelektronika. Uspekhi Sovremennoy Radioelektroniki, No. 8, 1999, pp. 30-44.

[2] I. Y. Gatilova, V. A. Pon'kin and T. S. Uzhakhov, "Determination of the Spatial Structure of Local Reflectors on the Surface of an Object from the Backward Scattering Diagram," Radio and Communications Technology, Vol. 5, No. 6, 2000, pp. 71-76.

[3] S. N. Kutishchev, "Iterative Method for Reconstruction of Local Scattering Sources of an Object from the Amplitude Backscattering Diagram," Electromagnetic Waves and Electronic Systems, Vol. 12, No. 6, 2007, pp. 23-27.

[4] S. N. Kutishchev, "Reconstruction of the Parameters of Local Scattering Sources of an Object from the Amplitude Backscattering Diagram," Electromagnetic Waves and Electronic Systems, Vol. 14, No. 2, 2009, pp. 66-71.

[5] S. N. Kutishchev, "Reconstruction of the Phase Back-Scattering Diagram of an Object from the Amplitude Backscattering Diagram," Electromagnetic Waves and Electronic Systems, Vol. 14, No. 3, 2009, pp. 68-72.

[6] S. N. Kutishchev, "Reconstruction of the Local Scattering Sources and Phase Backscattering Pattern of a Cylindrical Object from the Amplitude Backscattering Pattern," Electromagnetic Waves and Electronic Systems, Vol. 14, No. 6, 2009, pp. 42-48.

[7] S. N. Kutishchev, "A Method for Reconstructing the Parameters of Local Scattering Sources of an Object from the Backscattering Pattern," Radiophysics and Quantum Electronics, Vol. 54, No. 12, 2012, pp. 828-832. doi:10.1007/s11141-012-9344-6

[8] M. V. Konyukov, "Phase Problem in Aperture Synthesis Systems," Radiophysics and Quantum Electronics, Vol. 26, No. 11, 1983, pp. 1041-1049. doi:10.1007/BF01034670

[9] V. K. Ablekov, P. I. Zubkov and A. V. Frolov, "Optical and Optoelectronic Information Processing," Mashinostroenie, Moscow, 1976. 
[10] G. P. Bolts, "Inverse Problems in Optics," Springer-Verlag, New York, 1980. doi:10.1007/978-3-642-81472-3

[11] L. T. Tuchkov, "Radar Characteristics of Aircraft," Radio i Svyaz', Moscow, 1985.

[12] E. A. Shtager and E. V. Chaevskii, "Scattering of Waves from Bodies of Complex Shape," SOV Radio, Moscow, 1974.

[13] L. R. Rabiner and B. Gold, "Theory and Application of Digital Signal Processing," Prentice-Hall, Englewood Cliffs, 1975.

[14] P. A. Bakut, A. D. Ryakhin, K. N. Sviridov and N. D.
Ustinov, "Possibility of Single-Valued Reconstruction of the Image of an Object from the Modulus of Its Spatial Spectrum," Optics and Spectroscopy, Vol. 58, No. 4, 1985, pp. 552-554.

[15] I. N. Bronshtein and K. A. Semendyaev, "Handbook of Mathematics for Engineers and Students of Technical Colleges," Nauka, Moscow, 1980.

[16] E. V. Zakharov and Y. V. Pimenov, "Numerical Analysis of Diffraction of Radio Waves," Radio i Svyaz', Moscow, 1982. 